\title{
Die Winkelstabilität bei modernen Marknagelsystemen
}

\author{
Jochen Blum, Pol Maria Rommens
}

\section{Zusammenfassung}

Die Implementierung der Winkelstabilität bei Marknagelsystemen ist im Vergleich zu winkelstabilen Plattensystemen noch eher ein Novum. In der klassischen Philosophie der Marknagelung steht die elastische Osteosynthese an exponierter Stelle. Die Rigidität winkelstabiler Systeme scheint hier bei oberflächlicher Betrachtung einen Widerspruch zu provozieren. Moderne Marknagelsysteme weisen heute zumindest teilweise winkelstabile Verriegelungskomponenten auf. Diese sollen einerseits im osteoporotischen Knochen eine höhere Haltkraft verleihen, andererseits sollen sie das Indikationsspektrum der Marknage- lung von den rein diaphysären Frakturen hin zu den metaphysären Frakturen erweitern. Dazu dienen winkelstabile Bolzen-Nagel-, Schrauben-Nagelund Spiralklingen-Nagel-Kombinationen. Bei den heute erhältlichen winkelstabilen Marknagelsystemen stehen jene zur Behandlung der proximalen Humerusfrakturen, proximalen und distalen Femurfrakturen und proximalen Tibiafrakturen im Vordergrund, bei anderen Lokalisationen besteht noch deutlicher Entwicklungsbedarf. Ob die Winkelstabilität bei Marknägeln wirkliche therapeutische Vorteile besitzt, erfordert allerdings noch eine Reihe klinischer wie biomechanischer Studien.

\section{Einleitung}

Die Entwicklung und schließlich klinische Einführung der Winkelstabilität in der Verbindung von Platten und Schrauben hat nicht nur der Plattenosteosynthese eine gewisse Renaissance beschert und $\mathrm{zu}$ einem Überdenken auch deren Techniken unter dem Gesichtspunkt der „biologischen Osteosynthese“ geführt. So wurden dadurch konzeptionelle Prozesse in der Marknagelung angestoßen, aber auch bereits vorhandene Entwicklungen mit Nachdruck vorangetrieben, die eben jene Winkelstabilität bei den intramedullären Implantaten integriert sehen möchten.

Ähnlich der Plattenosteosynthese werden die Grenzen der Stabilisierungspotenz der Marknagelung dann ganz besonders deutlich, wenn die Qualität

OP-JOURNAL 2004; 20: 54-63

(c) Georg Thieme Verlag KG Stuttgart · New York der Knochensubstanz an den Schnittstellen Implantat-Knochen ausgesprochen vermindert ist, wie wir dies bei der Osteoporose täglich erfahren können.

Dies betrifft vornehmlich den KnochenBolzen-Kontakt, bei dem unter dem Hintergrund der Osteoporose frühzeitige Auslockerungen die Stabilität des gesamten Komplexes Implantat-Knochen erheblich gefährden, die Knochenheilung verhindern und schließlich zu Pseudarthrosen und/oder Materialbruch führen können.

Wenn auch das eigentliche Dilemma an der Schnittstelle Knochen-Bolzen zu finden ist, ist es nahe liegend, analog der Philosophie der winkelstabilen Platten, an der Schnittstelle Implantat/Implantat die Stabilität zu erhöhen. Für beide Verfahren gilt, dass die heutigen Möglichkeiten der Stabilitätsverbesserungen direkt an besagter Schnittstelle Knochen/Bolzen bzw. Knochen/Schraube auch gerade unter biologischen Gesichtspunkten relativ limitiert sind. Die Verbundosteosynthese mit Knochenzementen ist anerkanntermaßen keine Routinelösung für osteoporotische Knochen, biologische Knochenersatzstoffe allerdings mögen vor allem im metaphysären Bereich zukünftige Lösungen anbieten.

Folglich richtet sich die Aufmerksamkeit in der Weiterentwicklung intramedullärer Implantate auf die Verankerung der Verriegelungsentitäten (Bolzen, Schrauben, Klingen etc.). Dies betrifft einerseits deren Kontaktflächen im Knochen, andererseits aber deren Verankerung im eigentlichen Marknagel selbst.

Der Wunsch einer relativ rigiden Verbindung an der Schnittstelle Marknagel/Verriegelungsimplantat steht scheinbar im Widerspruch zur ursprünglichen Philosophie der elastischen Marknagelung. Dies mag auch erklären, warum die Entwickler neuer Marknagelsysteme so zögerlich mit dem Konzept der Winkelstabilität umgingen.

Das ursprüngliche Konzept Gerhard Küntschers sah im Rahmen der elastischen Verklemmung des Nagels in der Markhöhle bei diaphysären Frakturen keine Bolzen vor. In diesem klassischen Konzept beruht die Stabilisierungspotenz alleine auf dem direkten Kontakt des Nagels mit der Innenwand der Markhöhle (Abb.1). Der Begriff der Winkelstabilität lässt sich hierbei nicht anbringen. Diaphysäre Frakturen dies- und jenseits des Isthmus und damit verbundene Stabilitätsprobleme führten zunächst erst zum Konzept der aufgebohrten Marknagelung, wobei durch Vergrößerung der Kontaktfläche Nagel/Knochen das Spektrum damit behandelbarer Frakturen erweitert wurde. Allerdings erbrachte erst die Einführung der Verriegelungsmarknagelung eine deutliche Erweiterung der Einsatzmöglichkeit in den proximalen und distalen diaphysären Bereich.

Im Konzept der ursprünglichen Verriegelungsmarknagelung wurde bewusst als 


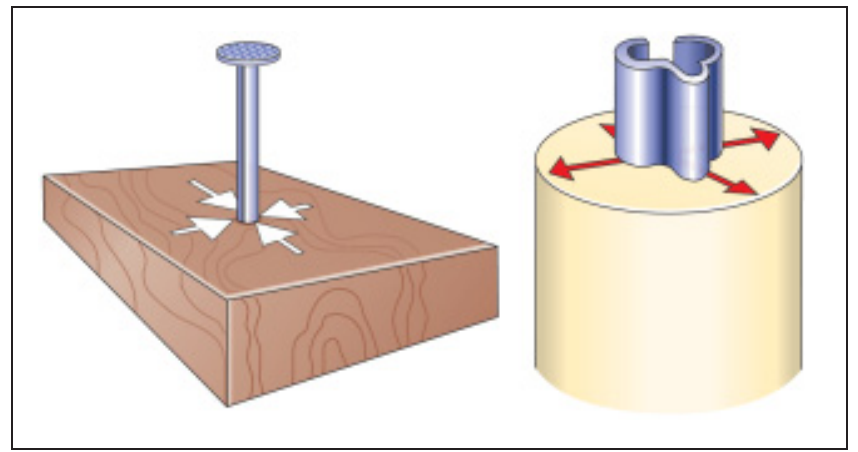

„Verriegelungsmedium“ nicht die Schraube, sondern der Bolzen verwendet (Abb.2). Somit besitzen diese gängigen Marknägel anstelle eines Gewindeloches eine glattwandige Bohrung, welche in der Regel einen, im Vergleich zum Bolzen selbst deutlich größeren Durchmesser aufweist. Dahinter steht die eigentliche Absicht, den Bolzen als Garant der Rotationssicherung und gegen Verkürzung oder Verlängerungstendenzen der Osteosynthese einzusetzen. Die ureigene Stabilität der Konstruktion ist weiterhin im Nagel selbst begründet, die damit verbundene Elastizität ist ausdrücklich erwünscht und wird als wesentliche mechanische Voraussetzung für die Entwicklung einer kallusreichen Knochenheilung

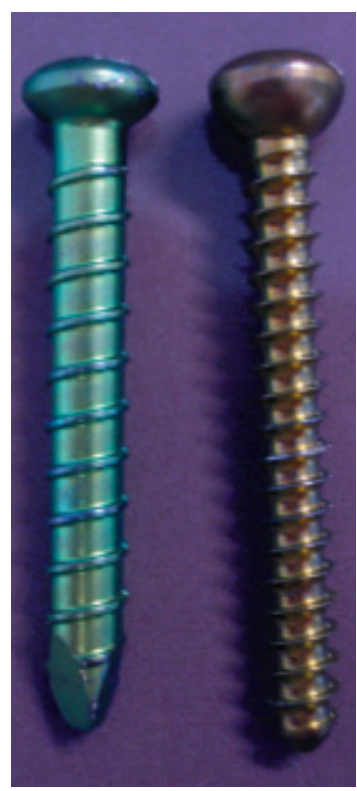

Abb. 2 Schraube und Bolzen: Schrauben (rechts) besitzen ein Gewinde, welches dem Gewinde z.B. in einer passenden Platte entspricht. Bolzen hingegen sind entweder glattwandig oder wie hier geriffelt (links).
Abb.1 Elastische Verklemmung: reziprok zu dem im elastischen Holz eingeschlagenen rigiden Nagel (links) verklemmt sich der elastische Nagel im rigiden Rohr (rechts). angesehen. Somit bestand bei aufgebohrten, mit hohen Durchmessern versehenen Marknägeln, welche mit relativ materialstarken Bolzen verriegelt wurden, weniger Anlass, über ein Konzept der Winkelstabilität nachzudenken.

Die aus biologischen Gründen Anfang der 90er-Jahre einsetzende rasante Entwicklung und der klinische Einsatz von „unaufgebohrten Marknagelsystemen“ hat im Kontext einer zeitgleich zunehmenden Frequenz immer älter werdender osteoporotischer Patienten die Aufmerksamkeit von Unfallchirurgen wie auch Implantatentwicklern mehr und mehr auf die kritische Schnittstelle „KnochenBolzen-Marknagel“ gelenkt. Die Verminderung der Kontaktfläche „Nagel-Knocheninnenfläche im Markraum“ bringt einen deutlichen Belastungszuwachs auf die eigentlichen Bolzen, die Verringerung des Nageldurchmessers erzwingt auch kleinere Bolzendurchmesser. Im Gegenzug kommt der damit in Verbindung stehenden Schnittstelle „Knochen-Bolzen“ für die gesamte Stabilisierungsfunktion wesentlich mehr Bedeutung zu. Damit verbunden ist neben einer erhöhten Bolzenbruchrate auch eine erhöhte Wahrscheinlichkeit der Bolzenlockerung im
Knochen. Kontinuierliches Gleiten des Nagels auf seinen Bolzen in horizontaler wie auch vertikaler Ebene überträgt sich auch an den Kontaktstellen des Bolzens mit dem Knochen und kann das dortige Auslockern unterstützen (Abb.3).

Die Technik mancher Unfallchirurgen, bei der Verriegelungsbohrung bewusst nicht das Nagelloch zentral-orthograd zu treffen, sondern durch einen eher schrägen Durchgang durch das Bolzenloch zu einer gewünschten Verklemmung des Bolzens zu gelangen, ist Ausdruck im Bemühen um höhere Stabilität an der Schnittstelle Nagel-Bolzen.

\section{Winkelstabile Marknagelsysteme}

Analog der Platte kann der Marknagel selbst nicht winkelstabil sein, sondern das Marknagelsystem, welches je nach Nageltyp beispielsweise die Bolzen beinhaltet. Die Winkelstabilität bei Plattensystemen ergibt sich auch erst aus ihrer Kombination der Platte mit Schrauben oder durch Sonderformen wie beispielsweise Winkelplatten, also einer Plattenkomponente die nicht nur dem Knochen anliegt, sondern ihn penetriert.

Die Verankerung der Verriegelungsimplantate im Nagel könnte somit winkelstabil sein. Da allerdings diese Verriegelungsimplantate, wie beispielsweise die Bolzen, in unregelmäßigen Abständen zur Nagelbasis in Verbindung mit dem Nagel gebracht werden, scheint bisher vor allem eine topographisch-partielle Winkelstabilität verfolgt worden $\mathrm{zu}$ sein. Hierunter ist die Applikation winkelstabiler Charakteristiken auf nur einen Teil dieser Verriegelungsimplantate (Bolzen, Schrauben, Spiralklingen etc.) zu verstehen. Die Begründung für dieses Vorgehen ist allerdings nicht wirklich wissen-

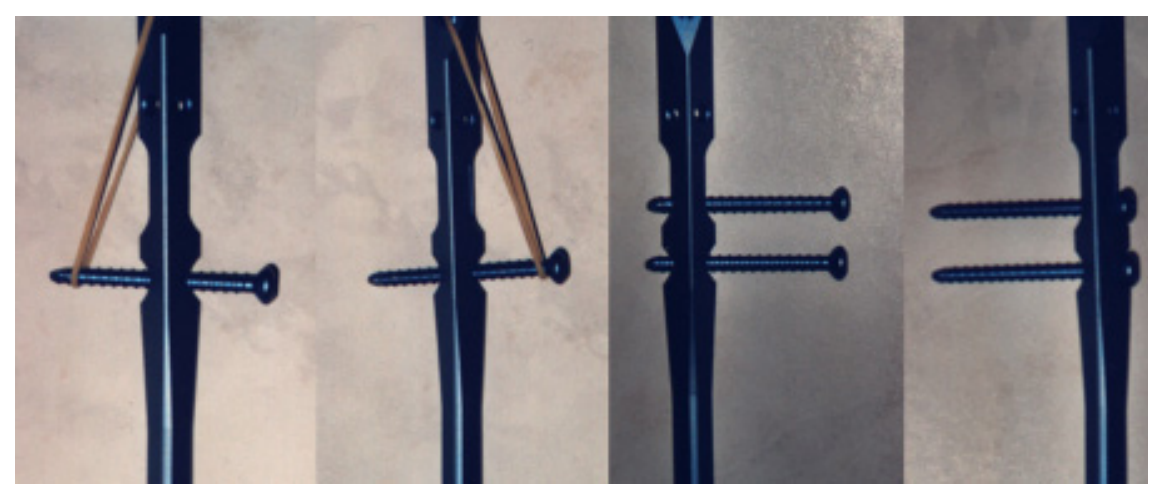

Abb. 3 Beispiel UTN: Kontinuierliches Gleiten des Nagels auf seinen Bolzen in horizontaler wie auch vertikaler Ebene überträgt sich auch an den Kontaktstellen des Bolzens mit dem Knochen und kann das dortige Auslockern unterstützen. 


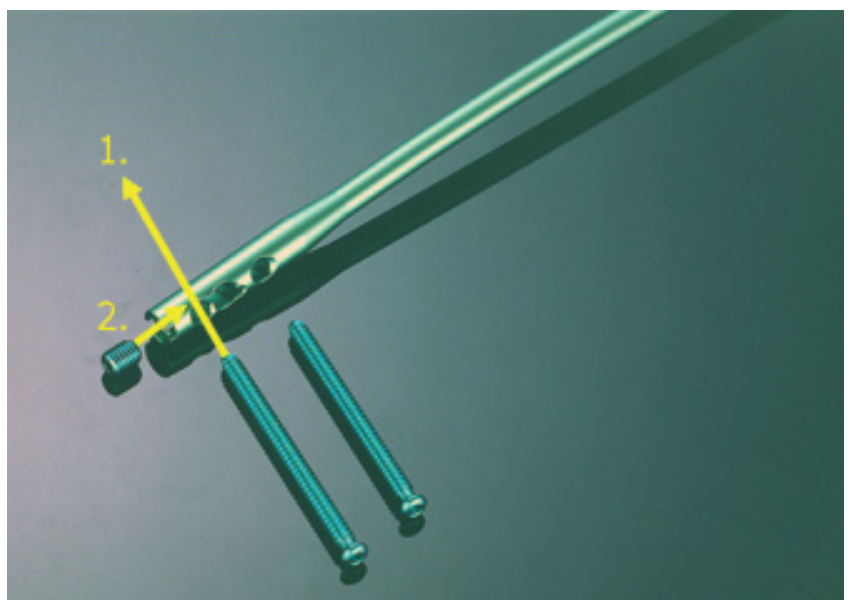

Abb. 4 Die Endkappe des Nagels dient neben ihrer Aufgabe, die Nagelbasis und deren Innengewinde vor Knocheneinwuchs zu schützen, vor allem dazu, Druck auf den basisnahen Bolzen zu bringen und inn winkelstabil zu verblocken - Beispiel DFN.
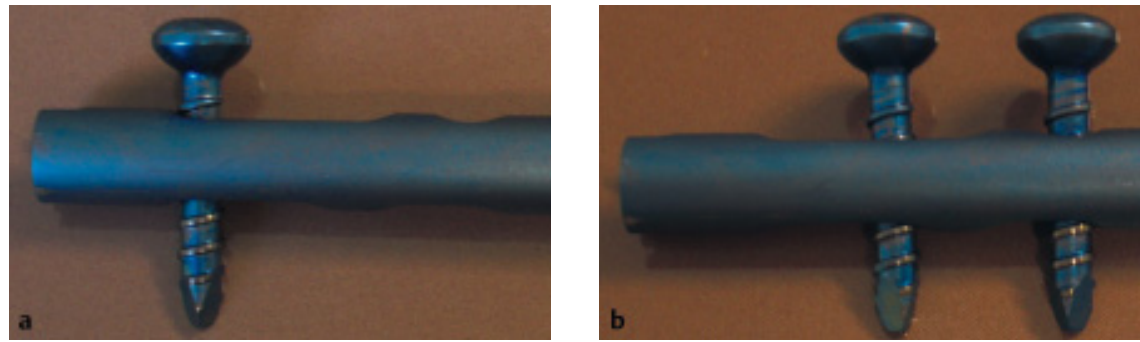

Abb. 5 Beispiel UHN: Der Bolzen im dynamischen Verriegelungsschlitz (a) wird mit dem daran fixierten Knochenanteil zum Frakturspalt hin verschoben und bewirkt dort interfragmentäre Kompression, verbiegt und verklemmt sich gegebenfalls. Der Effekt wird dann durch einen weiteren statischen Bolzen gesichert (b).

schaftlich, sondern fußt meist auf konstruktionstechnischen Zwängen. Dennoch werden auch Argumente hervorgebracht, die ihren Ursprung in der historisch gewachsenen Marknagelphilosophie begründet finden. So wird bei kompletter Winkelstabilität eine zu große Rigidität des gesamten Marknagelsystemes befürchtet, die im Widerspruch zur Forderung nach elastischer Marknagelosteosynthese stehen könnte. Allerdings fehlen hier klare Studienergebnisse, die uns einen klinisch verwertbaren Anhalt geben könnten, ab welcher Stabilität wir beispielsweise durch komplette Winkelstabilität ein Hemmnis der Knochenheilung kreieren.

\section{Prinzipien der Winkelstabilität bei Marknagelsystemen}

\section{Verriegelungsbolzen}

Je nach Verriegelungskomponente bieten sich unterschiedliche Prinzipien zum Erzielen von Winkelstabilität an.

Die „klassische“ Komponente ist der Bolzen. Das Konzept Küntschers versteht unter einem Bolzen im Gegensatz zum Nagel einen Metallzylinder, der in eine vor- gegebene Öffnung eingebracht wird, wobei er den gleichen Durchmesser besitzt, sich aber nicht verklemmt. Dies muss unter dem frühen Nagelkonzept gesehen werden, wo der Nagel einen größeren Durchmesser als die vorgegebene Öffnung besitzt und sich verklemmt (Abb.1). Es geschieht auf Basis der Einschlagkraft und der Tatsache, dass sich ein geschlitztes Rohr im Durchmesser anpassen kann.

Heutige Bolzen besitzen zwar eine Riffelung, aber kein echtes Gewinde (Abb.2). Somit ist die passende Bohrung im Nagel entsprechend glatt. und Nagel Winkelstabilität herzustellen, ist die Kompression des Bolzens in dem Moment, in dem er seine definitive gewünschte Position erreicht hat. Dies wird durch eine Druckschraube bewirkt, die über einen Gewindegang im Nagelinneren eingebracht wird und senkrecht auf den Bolzen Druck ausübt und mit dem Nagel verklemmt. Diese Druckschraube wird in einigen Nagelsystemen definitiv eingesetzt, sie verbleibt somit so lange im System, bis ggf. der gesamte Marknagel entfernt wird (Abb.4). Alter-
Eine realistische Lösung zwischen Bolzen nativ wird bei einigen Kompressionsnägeln dieser Druck nur temporär erzeugt, der betroffene Bolzen dabei bewusst deformiert, in der Art, dass er sich plastisch verformt und dadurch im Bolzenloch verklemmt. Einige Nagelsysteme sehen hier die Ergänzung durch einen zweiten statischen Bolzen vor, der das Kompressionsergebnis stabilisiert, bevor die komprimierende Druckschraube wieder entfernt wird (Abb.5) [1,2,3].

Die primäre oder sekundäre dynamische Verriegelung ist auch heute ein unumstrittenes Konzept bei diaphysären Quer- und kurzen Schrägfrakturen unter primär axialer Belastung. Das dynamische Prinzip muss nicht im Widerspruch zur Winkelstabilität stehen, wenn sie den dynamischen Bolzen ausklammert und nur auf die restlichen proximalen und distalen Bolzen angewandt würde.

\section{Verriegelungsschraube}

Im Gegensatz zu den Bolzen besitzen Schrauben ein echtes Gewinde (Abb.2). Einige Hersteller setzen Schrauben auch bei Marknagelsystemen ein, die allerdings im Nagel kein Gegengewinde aufweisen, sondern dort auch wie bei den Bolzen durch eine glatte Bohrung hindurch treten. Diese Systeme sind natürlich nicht winkelstabil. Man verspricht sich in erster Linie eine bessere Fixierung der Schraube im Knochen selbst.

Um bei Verriegelungsschrauben Winkelstabilität zu erreichen, sind mehrere Prinzipien denkbar, aber nicht allesamt im klinischen Alltag praktikabel.

Analog der bewährten winkelstabilen Schrauben-Plattenverbindung könnte ein dem Schraubenkopf eigenes Gewinde dem im Verriegelungsloch des Nagels integrierten entsprechen. Dieses Prinzip erscheint aber unrealistisch und schwer umsetzbar, da einerseits die zugewandte Kortikalis relativ groß gebohrt werden müsste, um den Schraubenkopf hindurch bringen zu können. Dazu kommt, dass somit nur unikortikale Schraubenverriegelungen möglich wären. Die damit verbundene Stabilitätsminderung steht nicht im Verhältnis zum Gewinn durch Winkelstabilität. Eine später erwünschte Entfernung dieser Schraube wäre kaum möglich, da das ursprüngliche Eintrittsloch zwischendurch wieder knöchern verschlossen wäre.

Denkbar ist es, das Kompressionsprinzip, wie es bei Bolzen angewendet wird, auch 

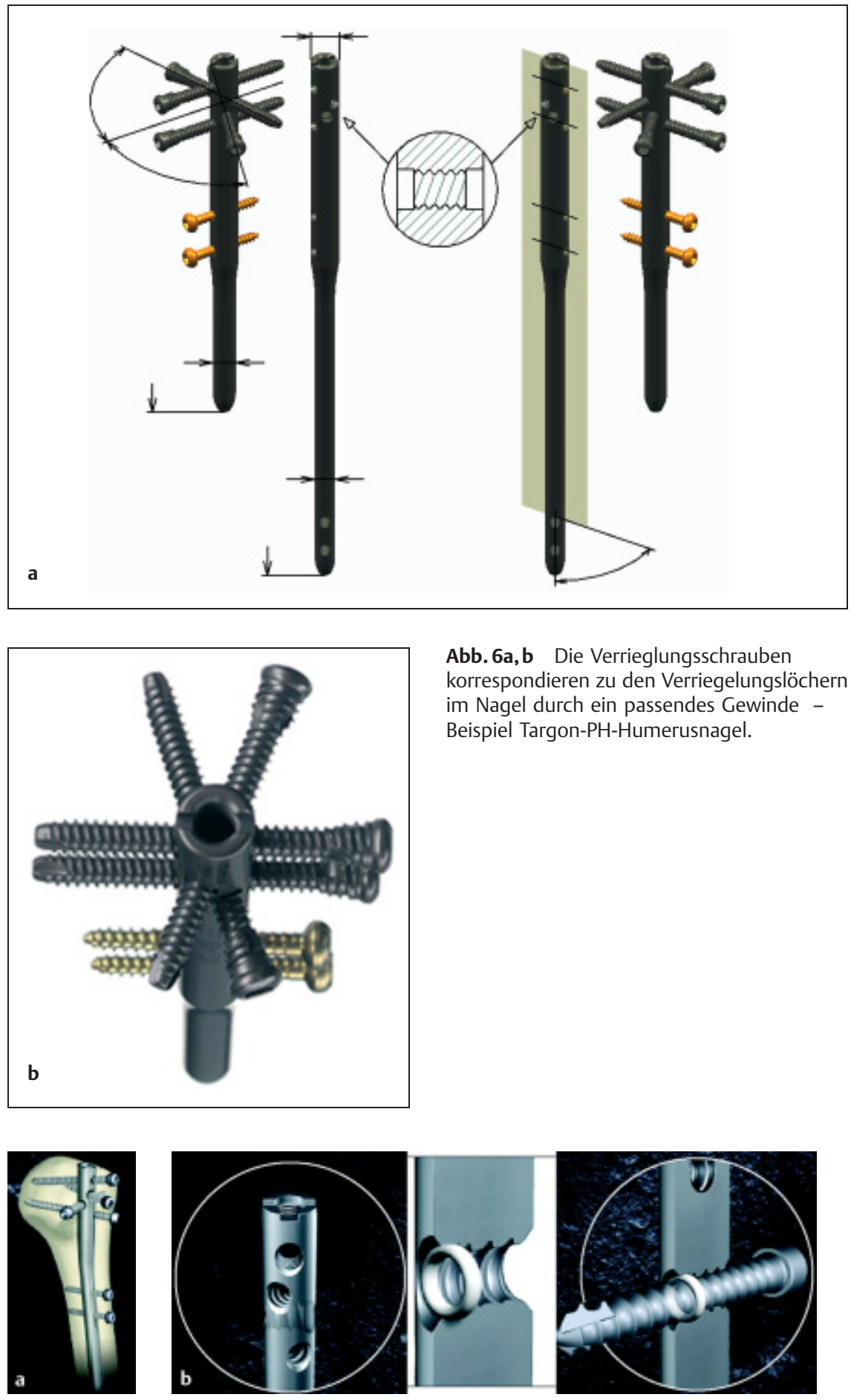

Abb. 7a, b Zusätzliche Integration von Kunststoffringen in das Gewinde des Nagels mit der Absicht, eine bessere Haltkraft der winkelstabilen Verriegelungsschraube im Nagel zu erzielen Beispiel T2-PHN.

bei Verriegelungsschrauben zu nutzen. Ob die gewindetragende Oberfläche der Schraube im Gegensatz zum Bolzen hierbei Vor- oder Nachteile besitzen würde, bleibt zu untersuchen.
Abb.6a, b Die Verrieglungsschrauben korrespondieren zu den Verriegelungslöchern im Nagel durch ein passendes Gewinde Beispiel Targon-PH-Humerusnagel.
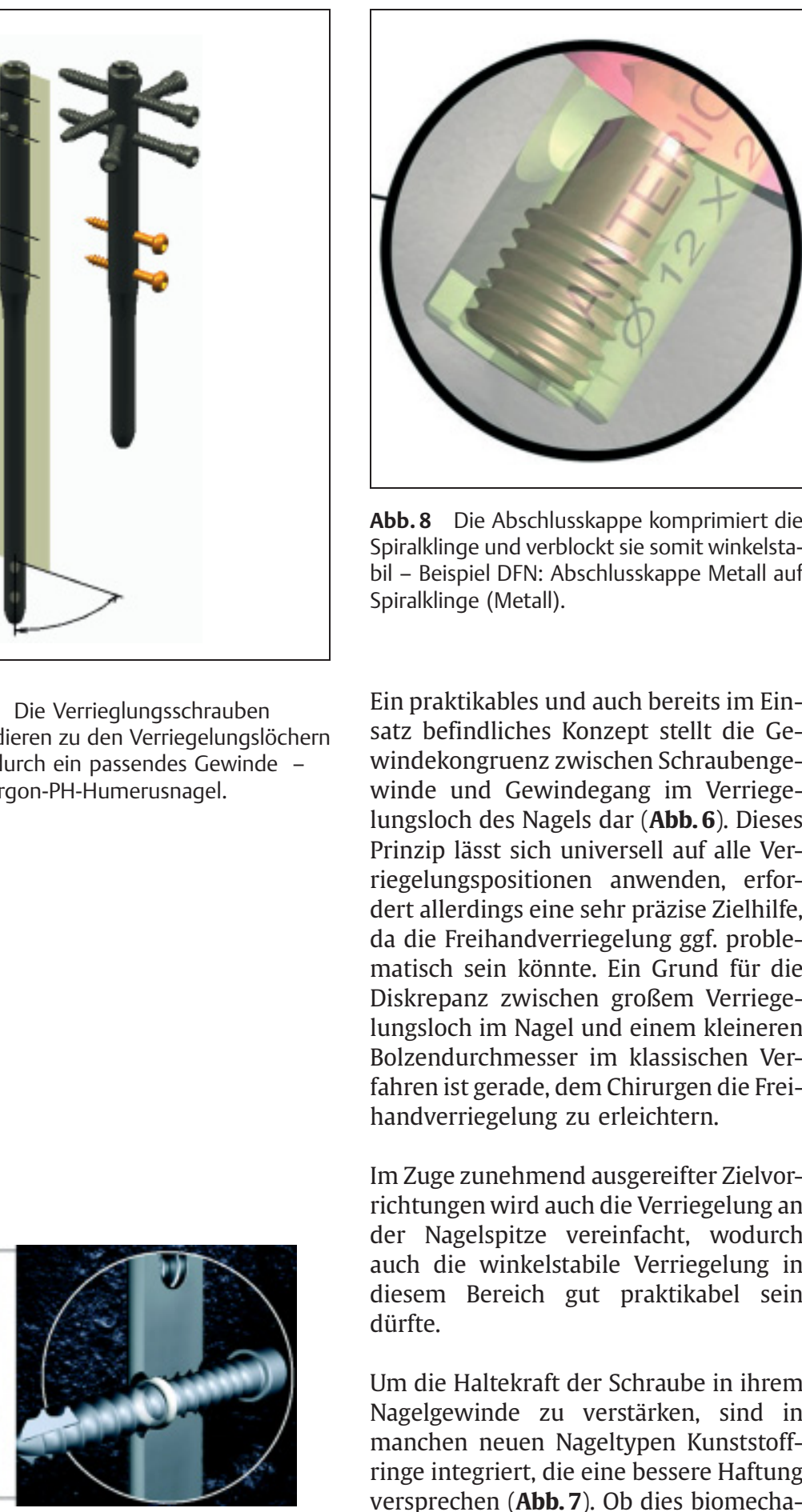

Abb. 8 Die Abschlusskappe komprimiert die Spiralklinge und verblockt sie somit winkelstabil - Beispiel DFN: Abschlusskappe Metall auf Spiralklinge (Metall).

Ein praktikables und auch bereits im Einsatz befindliches Konzept stellt die Gewindekongruenz zwischen Schraubengewinde und Gewindegang im Verriegelungsloch des Nagels dar (Abb.6). Dieses Prinzip lässt sich universell auf alle Verriegelungspositionen anwenden, erfordert allerdings eine sehr präzise Zielhilfe, da die Freihandverriegelung ggf. problematisch sein könnte. Ein Grund für die Diskrepanz zwischen großem Verriegelungsloch im Nagel und einem kleineren Bolzendurchmesser im klassischen Verfahren ist gerade, dem Chirurgen die Freihandverriegelung zu erleichtern.

Im Zuge zunehmend ausgereifter Zielvorrichtungen wird auch die Verriegelung an der Nagelspitze vereinfacht, wodurch auch die winkelstabile Verriegelung in diesem Bereich gut praktikabel sein dürfte.

Um die Haltekraft der Schraube in ihrem Nagelgewinde $\mathrm{zu}$ verstärken, sind in manchen neuen Nageltypen Kunststoffringe integriert, die eine bessere Haftung versprechen (Abb. 7). Ob dies biomechanisch signifikant höhere Stabilität bietet, ist bisher nicht geklärt.

\section{Spiralklingen}

in wäre es möglich, mittels konischer Verklemmung der Schraube im Verriegelungsloch Winkelstabilität zu erzielen. Ein sicherlich nicht zu unterschätzendes Problem bleibt die Ungewissheit, wie im erforderlichen Falle ggf. diese Schrauben wieder entfernt werden könnten.
Anstelle von Bolzen und Schrauben werden bei verschiedenen Nagelsystemen auch Spiralklingen eingesetzt. Sie sollen im Gegensatz zum Bolzen eine bessere Abstützung und Haltekraft im spongiösen Knochen gewährleisten. Somit liegt hier die Hauptindikation bei metaphysä- 

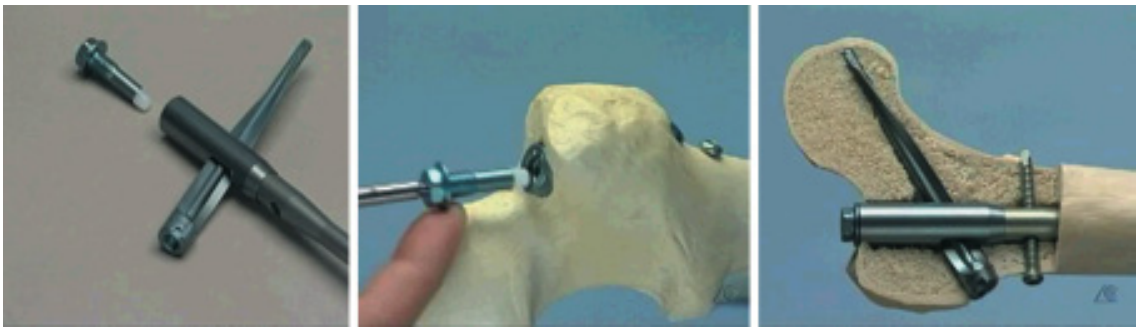

Abb.9 Beispiel UFN: Abschlusskappe Kunststoff auf Spiralklinge (Metall).

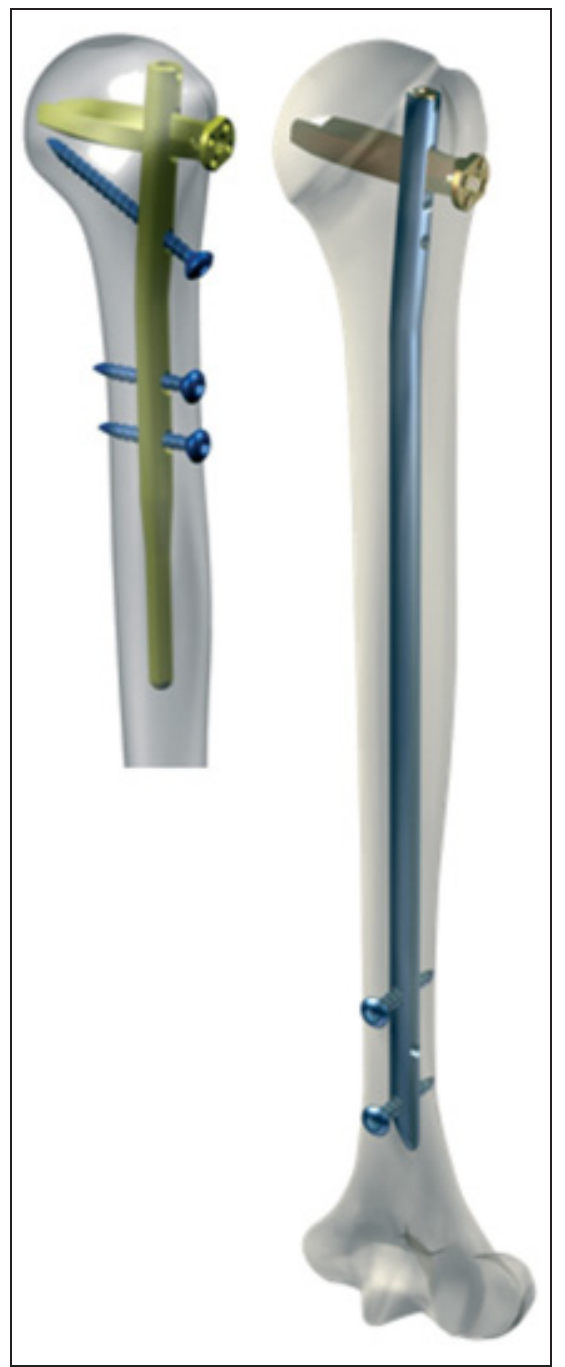

4 Abb.10 Proximaler Humerusnagel (AO) (links) und UHN (rechts), jeweils mit Spiralklinge zur Verriegelung im Humeruskopf.

ren Frakturen und beim osteoporotischen Knochen. Die Winkelstabilität ist unbedingter Bestandteil des Konzeptes, welche in der Regel über Kompression mit Druckschrauben erzielt wird. Oftmals lässt sich dies mit der Abschlusskappe der Nagelbasis erzielen.

Diese Kompression der Endkappe erfolgt entweder durch direkten Druck von Metall (Endkappe) auf Metall (Spiralklinge) (Abb.8) oder durch einen der Endkappe aufsitzende Kunststoffüberzug als Kunststoff-Metall-Kontakt (Abb. 9).

\section{Aktuelle Realisierung der Winkelstabilität bei gebräuchlichen Marknagelsystemen}

\section{Humerus}

Die Verriegelungsmarknagelung der Humerusschaftfraktur im antegraden wie auch retrograden Verfahren hat in den letzten Jahren eine zunehmende Bedeutung erlangt $[18,19]$. In der Behandlung reiner Schaftfrakturen sind hierbei Aspekte der Winkelstabilität lediglich auf experimenteller Ebene beachtet worden. Hingegen in der Marknagelung proximaler, metaphysärer Humerusfraktu-

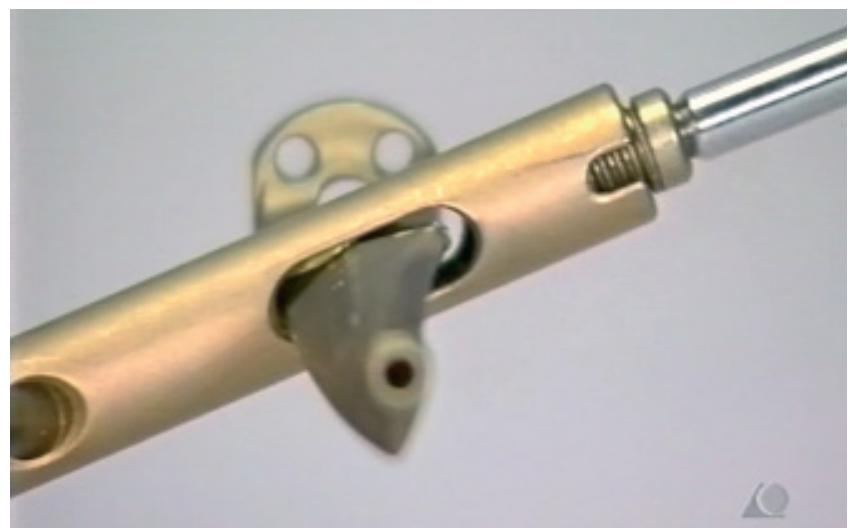

Abb.11 Die Endkappe des PHN komprimiert nach definitivem Einschrauben die Spiralklinge winkelstabil.

ren besitzen bereits mehrere Marknagelsysteme winkelstabile Komponenten. Andererseits ist die Verriegelungsmarknagelung distaler metaphysärer Humerusfrakturen eine generell bisher kaum beachtete Thematik, so dass hier auch bezüglich der Winkelstabilität keine nennenswerten Entwicklungen zu beobachten sind.

Sowohl der „Unaufgebohrte Humerusnagel (UHN)“ wie auch der „Proximale Humerusnagel (PHN)“ der AO bieten durch die Integration einer Spiralklinge an der Nagelbasis, welche durch eine Endkappe verblockt wird, dort Winkelstabilität an (Abb.10 und 11). Dies bleibt dem antegraden Verfahren vorbehalten und stellt beim PHN eine obligate, beim UHN eine fakultative Komponente dar (Abb.12 und 13) [4]. Biomechanische Studien bescheinigen dieser Konstruktion eine außerordentlich hohe Stabilität unter Druck-, Biege- wie auch Rotationsbelastung $[9,10,14]$. In Fällen extremer Osteoporose kann allerdings auch dadurch ein „Cut-out"-Phänomen beobachtet werden, bei dem die Klinge durch den weichen Humeruskopf schneidet. Eine aktuelle AO-Multicenter-Studie soll die Qualitäten des klinischen Einsatzes des PHNs klären.

Der proximale Humerusnagel Targon-PH $[15,20]$ besitzt in mehreren Ebenen Verriegelungsschrauben durch die Nagelbasis, welche auf ein entsprechendes Gegengewinde im Verriegelungsloch treffen (Abb.6).

Auf ähnlichem Prinzip fußt auch der T2PHN, bei dem allerdings zusätzliche Kunststoffringe in die Verriegelungslöcher und deren Gewinde integriert werden, um die Haltekraft der Schraube dort zu verstärken (Abb. 7).

\section{Unterarm}

Die Verriegelungsnagelung hat bei Radius- und Ulnafrakturen bei weitem nicht die Verbreitung gefunden, wie an Oberarm, Ober- und Unterschenkel. Das heute bekannteste Nagelsystem, der „Foresight-Nagel“, weist keinerlei winkelstabile Kriterien auf, sondern besitzt konventionelle Verriegelungsschrauben, welche durch ein glattes Verriegelungsloch in den Nagel eingebracht werden [22].

Verschiedene kurze Olecranonnägel, welche allerdings bisher nicht weit verbreitet sind, besitzen ebenfalls keine winkelsta- 

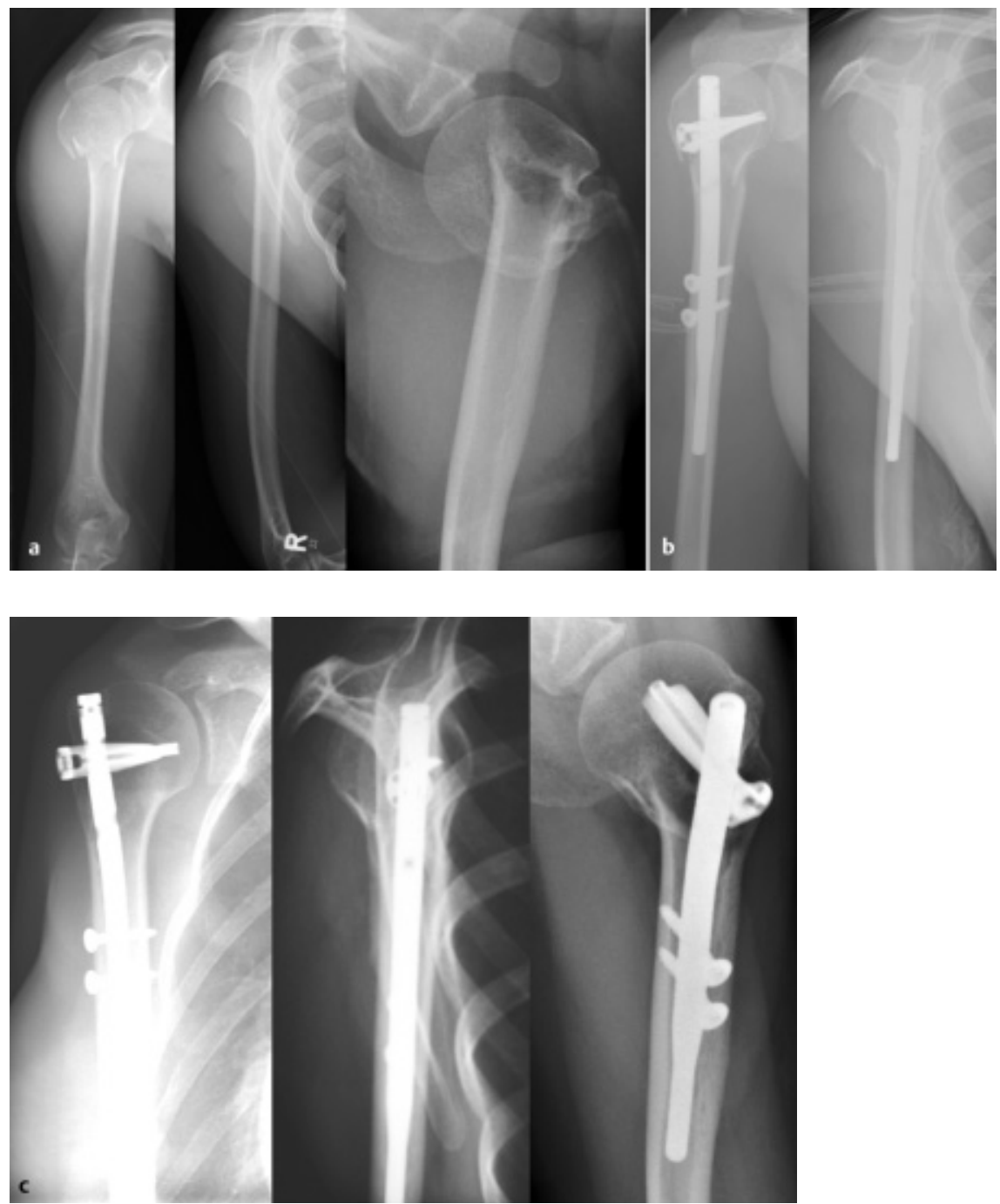

Abb.12 (a) Subcapitale Humerusfraktur -Unfallaufnahmen. (b) Versorgung mit PHN und Spiralklinge - postoperative Aufnahmen (c) Röntgenaufnahmen 3 Monate nach Operation mit Ausheilung der Fraktur.

bilen Charakteristiken. Der „XS-Olecranonnagel" sieht Kirschner-Drähte durch glattwandige Nagelperforationen zur mehrfachen Verriegelung vor [5].

\section{Oberschenkel}

Verriegelungsmarknagel-Systeme existieren in großer Zahl und auch technischen Varianten für metaphysäre, wie auch diaphysäre Femurfrakturen. Grundprinzipien der winkelstabilen Verriegelung sind in der Regel die Kompression entweder einer Spiralklinge oder eines Bolzens.

In der Palette der AO-Marknägel für das Femur besteht sowohl für den „Unaufgebohrten Femurnagel (UFN)“ wie auch für den „Distalen Femurnagel (DFN)“ die Option einer Spiralklinge zur Verriegelung der Nagelbasis. Beim DFN soll diese die Haltekraft des Systemes vor allem im osteoporotischen Knochen verbessern, beim UFN soll sie die Indikation des UFN von den rein diaphysären Frakturen hin zu den subtrochantären Frakturen erweitern. Die Winkelstabilität wird über die Kompression der Endkappe auf die Spiralklinge erzielt (Abb.9 und 14).

Bei regulärer Knochenqualität kann diese Verriegelung der DFN-Nagelbasis auch alleine durch Bolzen erfolgen, wo dann der distalste Bolzen ebenfalls mit einer Endkappe komprimiert wird und dadurch Winkelstabilität erhält (Abb.15 und 16) $[6,11,12,13,21]$.

Bei pertrochantären Femurfrakturen, welche mit dem neuen „Proximalen Femurnagel (PFN)“ versorgt werden kön- nen, stellt sich die Frage, ob hier die Winkelstabilität mit Kompression der Spiralklinge im Widerspruch zu dem sonst geforderten Prinzip der dynamischen Fixierung steht.

\section{Unterschenkel}

Die Verriegelungsmarknagelung bei Tibiafrakturen ist in der Regel den diaphysären Frakturen vorbehalten. Neuentwicklungen zeigen jedoch, dass auch die metaphysären Regionen der Tibia, sowohl proximal als auch distal, „nagelbar“ und „verriegelbar“ sind. Dies erforderte sowohl ein neues Nageldesign als auch eine neue Geometrie der Verriegelungsanordnung, aber auch neue Bolzentypen. Das Prinzip der Spiralklinge hat bisher an der Tibia nicht die Bedeutung wie an Humerus und Femur erlangen können.

Das neue, noch in der Erprobung befindliche Tibianagelsystem (TNS) der AO (Abb.17), welches aus einem „Proximalen Tibianagel (PTN)“ weiterentwickelt wurde (Abb.18 und 19), integriert die Winkelstabilität durch Kompression des proximalsten Bolzens mittels einer Endkappe. Biomechanische Studien bescheinigen dieser neuen proximalen Bolzenanordnung sehr hohe Stabilität $[7,8]$.

Neu ist das Prinzip der Winkelstabilität eines Bolzens im Tibianagel nicht. Speziell für Quer- und kurze Schrägbrüche der Tibia wurden bereits in den 80er-Jahren Tibiakompressionsnägel entwickelt. Durch Kompressionsschrauben, welche Druck auf einen basisnahen Bolzen ausüben, wurde einerseits die Fraktur komprimiert, andererseits aber auch der betreffende Bolzen winkelstabil verblockt bzw. sogar durch weitere Deformierung im Verriegelungsloch verklemmt $[3,16,17]$.

\section{Diskussion}

Marknagelsysteme bieten im Vergleich zu Platten und Schrauben in der Regel eine reduzierte Zugangsmorbidität und den Vorteil der günstigeren Biomechanik als zentraler Lastträger. Neuentwicklungen scheinen sie sogar für metaphysäre Frakturen interessant zu machen, wohin gehend sie bei den epiphysären Frakturen weiterhin eine eher fragwürdige Rolle einnehmen. Da aber gerade bei der zunehmenden Osteoporose unserer älteren Patienten Platten wie Nägel gleichermaßen an die Grenzen ihrer Stabilisierungspotenz geraten, ist es sicherlich günstig, von den Neuentwicklungen winkelstabi- 

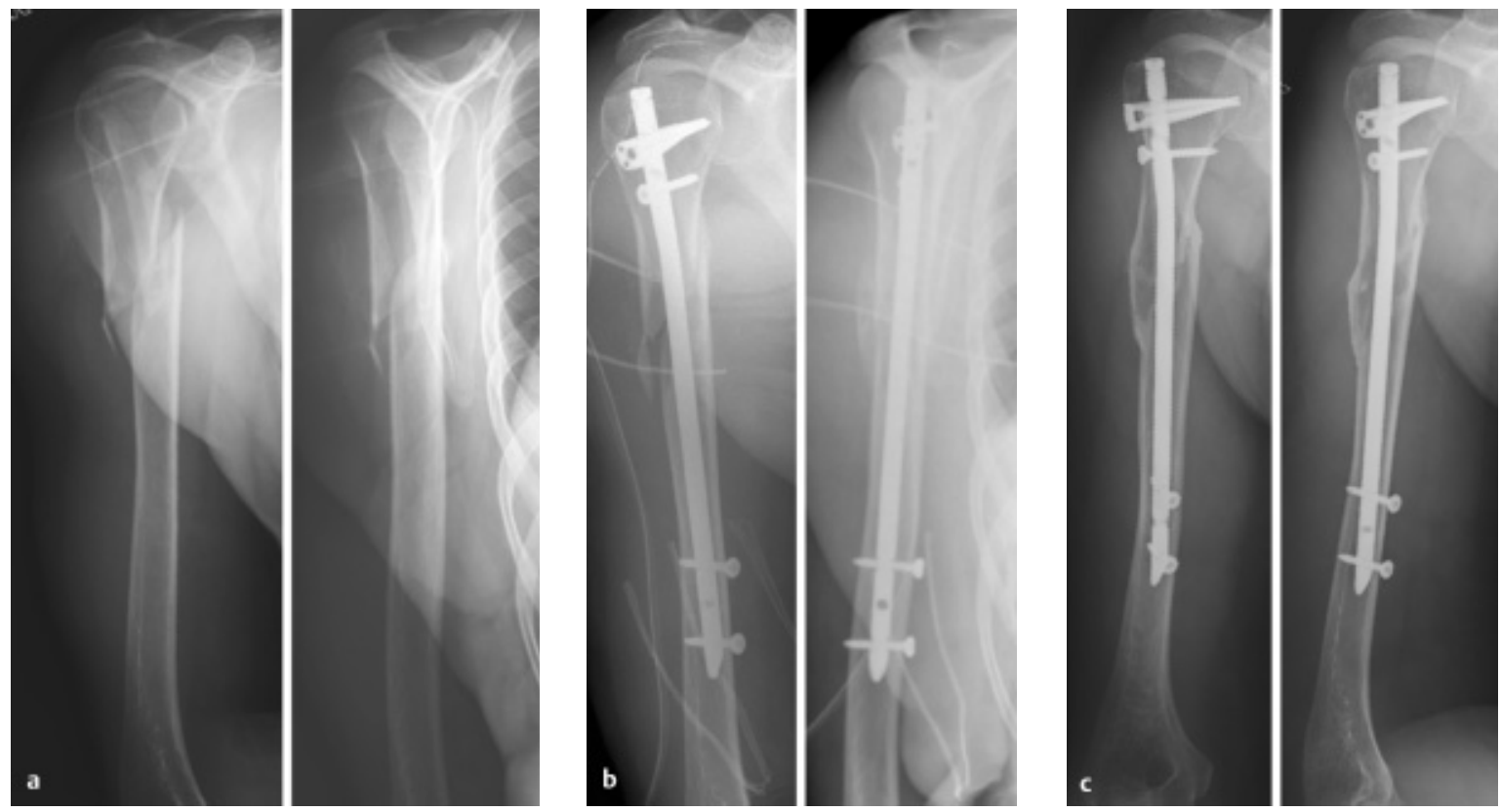

Abb.13 (a) Proximale Humerusschaftspiralfraktur mit Ausläufer in das Tuberculum majus - Unfallaufnahmen, (b) Versorgung mit P7,5 mm-UHN und Spiralklinge - postoperative Aufnahmen, (c) Röntgenaufnahmen 5 Monate nach Operation mit Ausheilung der Fraktur.
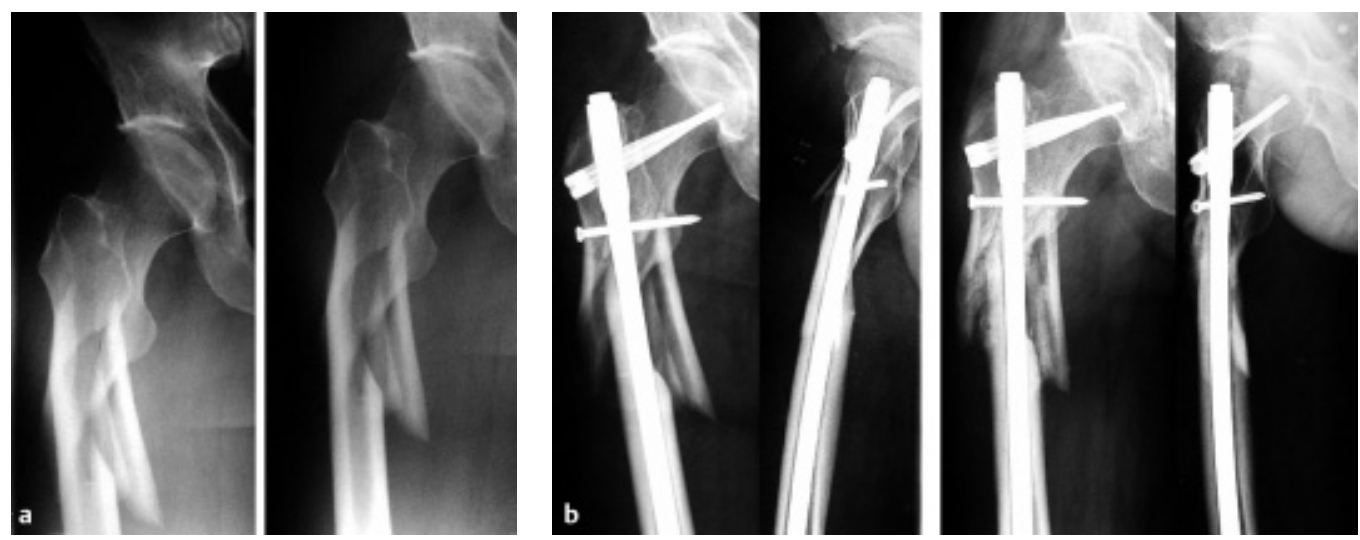

Abb.14 (a) Subtrochantäre Femurfraktur-Unfallaufnahmen, (b) Versorgung mit UFN und Spiralklinge - postoperative Aufnahmen (links) und 2 Monate nach Eingriff (rechts), (c) Röntgenaufnahmen 4 Monate und 1 Jahr nach Operation mit Ausheilung der Fraktur.

ler Plattensysteme zu lernen. Die stabilere Verankerung der Schrauben im Plattenloch ist sicherlich auch auf jene zwischen Bolzen/Schraube/Spiralklinge und Nagel übertragbar.

Interessanterweise hat man sich hier bisher aber weitgehend mit Teillösungen zufrieden gegeben. Bei Betrachtung der gegenwärtigen Nagelsysteme, welche winkelstabile Komponenten besitzen, wird die Winkelstabilität in Regel nicht auf das ganze Implantat ausgedehnt. So findet man meist eine winkelstabile Verriegelung im nagelbasisnahen Anteil, hingegen im Bereich der Nagelspitze nicht. Auf die technischen Hintergründe dieser Beschränkung wurde hingewiesen. Eigene biomechanische Experimente deuten aber darauf hin, dass gerade bei sehr weiten Markhöhlen im Zusammenhang mit Osteoporose, eine Winkelstabilität auch jener Bolzen erwünscht wäre, die auf der Seite der Nagelspitze platziert werden. Dies würde ein horizontales Gleiten des Nagels auf diesen Bolzen verhindern.

Die bittere Erfahrung jener Chirurgen, die erfolgreich mit winkelstabilen Platten arbeiten, aber in Fällen mit extremster Osteoporose mit Ausrissen der Platte ge- rade aufgrund deren hoher Stabilität an der Schnittstelle Schraube/Platte konfrontiert sind, wird natürlich auch für die Anwender winkelstabiler Nägel eintreten. Somit lässt sich der effektive Gewinn durch eine Winkelstabilität bei Marknagelsystemen bisher nicht eindeutig bestimmen. Hier ist sowohl klinische als auch biomechanische Forschung notwendig. Bisherige klinische Studien mit partiell winkelstabilen Marknagelsystemen konnten den wirklichen Anteil der Winkelstabilität an der Qualität, Vollständigkeit und Geschwindigkeit der knöchernen Heilung, wie auch des funktionellen Ergebnisses, nicht herausarbeiten. 

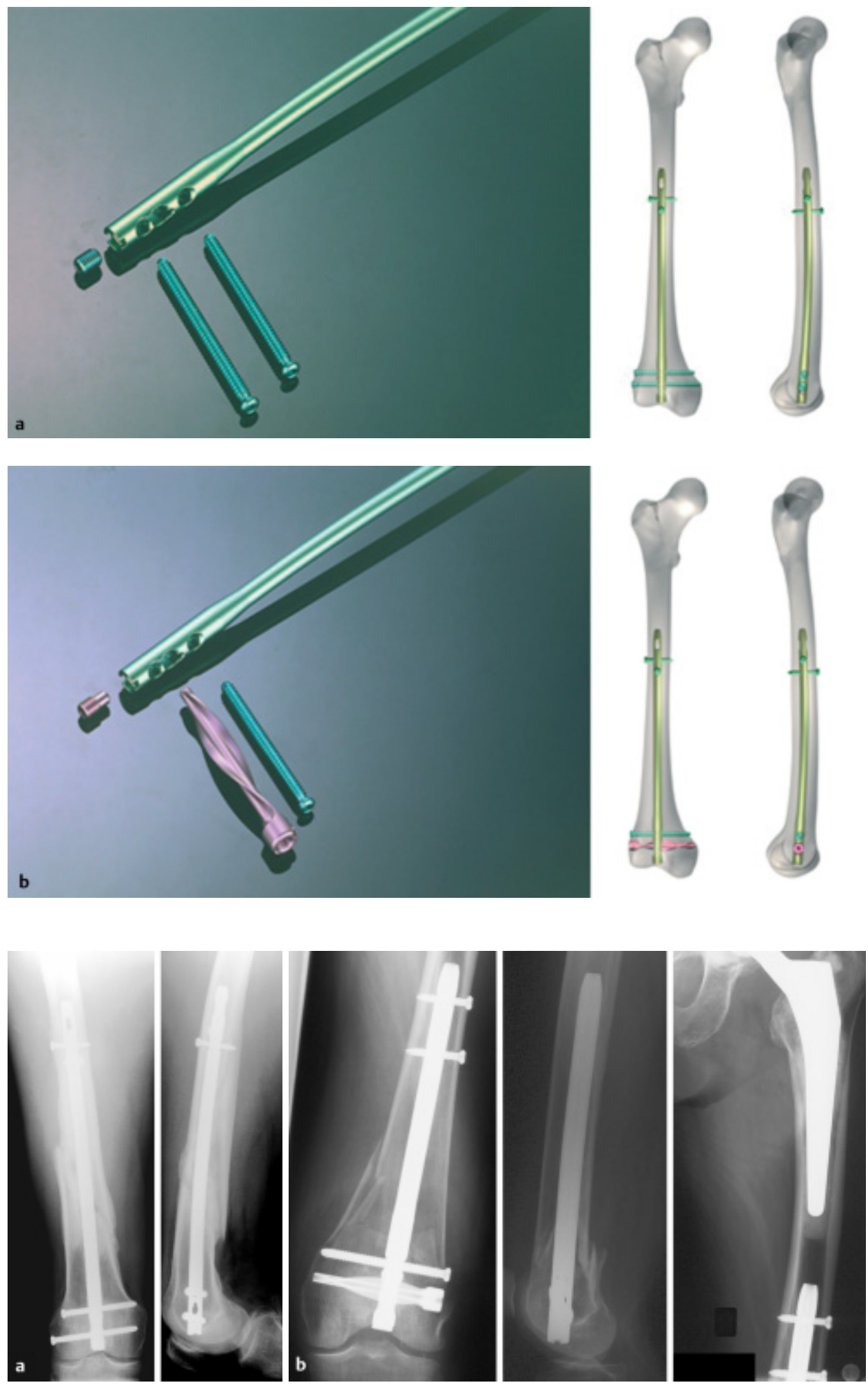

Interessanterweise lässt das Design biomechanischer Untersuchungen solcher neuen Nagelsysteme auch selten wirklich erkennen, welchen Anteil effektiv die Winkelstabilität an den Stabilitätsergebnissen besitzt. Es wird in der Regel nicht das gleiche Implantat mit winkelstabiler gegen nicht-winkelstabile Verriegelung geprüft, sondern das neue Nagelsystem gegen ein anderes, meist älteres Implantat.

Neben diesen biomechanischen Untersuchungen wird dann noch das Tierexperi-
Abb.16 (a) Klinisches Beispiel einer ausgeheilten Fraktur versorgt durch DFN mit winkelstabiler Verblockung des distalsten Bolzen.

(b) Klinisches Beispiel einer ausgeheilten Fraktur versorgt durch DFN mit winkelstabiler Verblockung der Spiralklinge für osteoporotischen Knochen.

Abb. 15 (a) DFN mit winkelstabiler Verblockung des distalsten Bolzen. (b) DFN mit winkelstabiler Verblockung der Spiralklinge für osteoporotischen Knochen.

ment erforderlich, welches uns klare Hinweise geben muss, ob die Winkelstabilität bei Marknägeln deren Prinzip der elastischen Osteosynthese derart aushebelt, dass es sogar zu einer Behinderung der Knochenheilung kommen könnte. Allerdings muss man hier erkennen, dass 

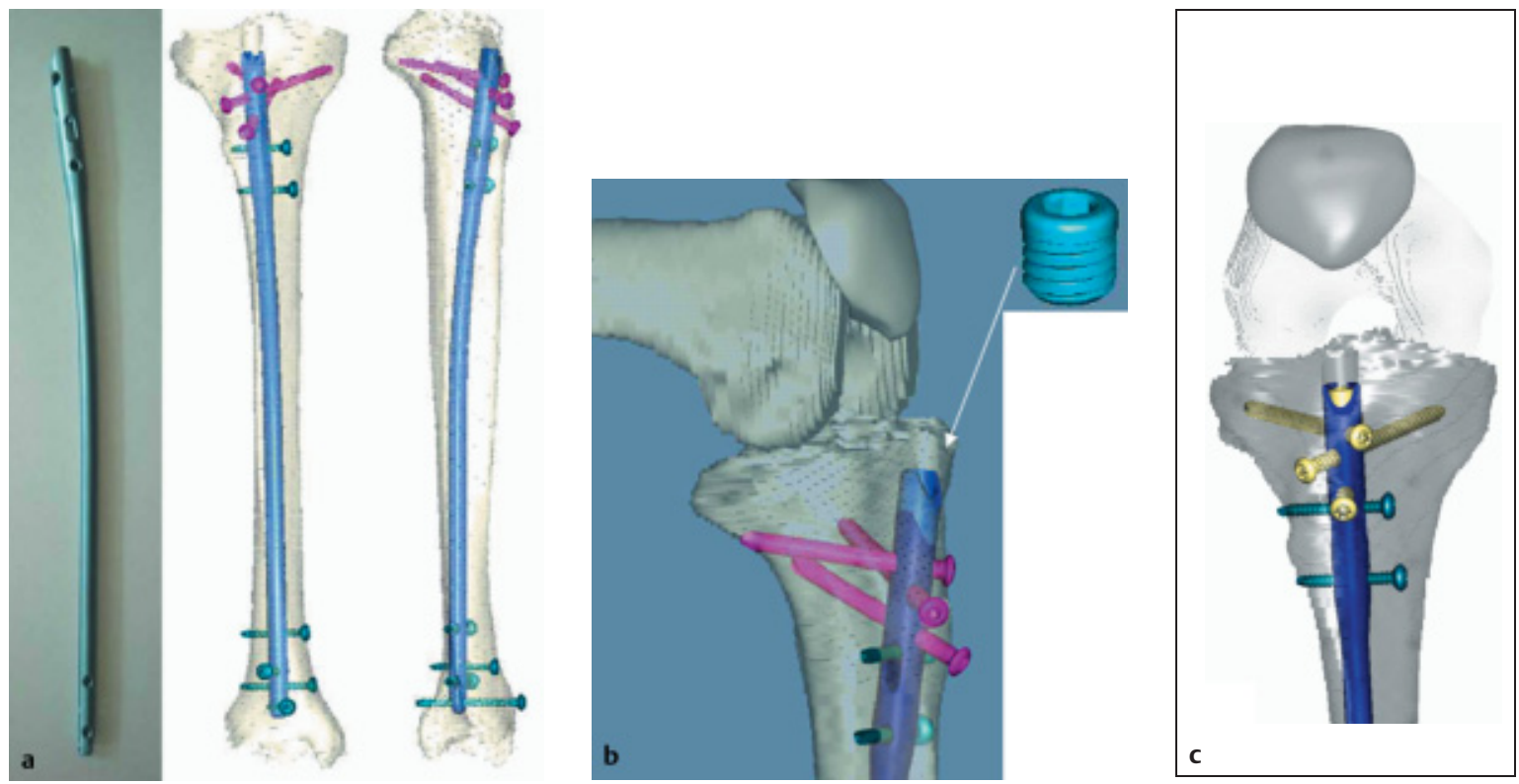

Abb.17 (a) Das neue Tibia-Nail-System (TNS) der AO zur Erweiterung des Indikationsspektrums von diaphysären zu proximalen und distalen metaphysären Tibiafrakturen. (b) Die Endkappe wird nach Verriegelung in die Nagelbasis eingedreht. (c) Durch Kompression auf den Basis-nahen Bolzen entsteht eine winkelstabile Verankerung.
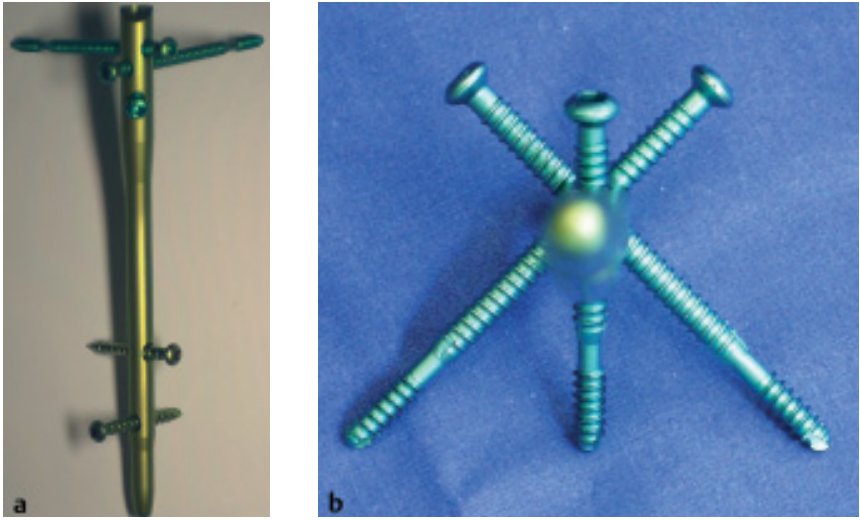

Abb.18 (a) Gleiches Prinzip beim Proximalen Tibianagel PTN für proximale metaphysäre Tibiafrakturen, (b) die Endkappe wird nach Verriegelung in die Nagelbasis eingedreht und verankert den Basis-nahen Bolzen dieser Tripod-artigen Verriegelungsanordnung winkelstabil.

durch die in der Regel sehr proximale wie auch distale Verriegelung zwar einerseits dort durch die Winkelstabilität eine generell hohe Stabilität des Implantates im Knochen gewährleistet wird, andererseits aber gerade in der Frakturregion keine starre, sondern im Idealfall eine ausreichend elastische Zone bestehen bleibt, die für eine kräftige Kallusformation verantwortlich zeichnen kann.

Die Weiterentwicklung winkelstabiler Marknagelsysteme wird demnach für Hersteller wie auch Unfallchirurgen eine der wichtigsten Herausforderungen der nächsten Jahre sein.

\section{Literatur}

${ }^{1}$ Blum J, Machemer H, Högner M, Baumgart F, Schlegel U, Wahl D, Rommens PM. Biomechanik der Verriegelungsmarknagelung bei Oberarmschaftfrakturen. Vergleichsuntersuchungen zweier Marknagelsysteme und des Effekts der interfragmentären Kompression beim unaufgebohrten Humerusnagel. Unfallchirurg 2000; 103: $183-190$

${ }^{2}$ Blum J, Rommens PM. Interfragmentäre Kompression bei der Humerusschaftnagelung mit einem speziellen Kompressionsgerät. Akt Traumatol 2001; 31: 90 -94

${ }^{3}$ Blum J, Rommens PM. Die komprimierte Marknagelung bei Oberarmschaftfrakturen. Trauma Berufskrankh 2001; 3: 188-194

${ }^{4}$ Blum J, Hessmann MH, Rommens PM. Behandlung proximaler metaphysärer Humerusfrakturen mit Verriegelungsmarknage- lung und Spiralklinge - erste Erfahrungen mit einem neuen Implantat. Akt Traumatol 2003; 33: 7-13

${ }^{5}$ Friedl W. Zuggurtungsnagel - Der XS- und XXS-Nagel in der Versorgung zugbelasteter und Gelenk- respektive gelenknaher Frakturen. Trauma Berufskrankh 2004;1: im Druck

${ }^{6}$ Grass R, Biewener A, Endres T, Rammelt S, Barthel S, Zwipp $\mathrm{H}$. Klinische Erprobung des distalen Femurnagels. Unfallchirurg 2002; 105: $587-594$

${ }^{7}$ Hansen M, Mehler D, Voltmer W, Rommens PM. Die proximale extraartikuläre Tibiafraktur. Unfallchirurg 2002; 105: 858-72

${ }^{8}$ Hansen M, Blum J, Voltmer W, Mehler D, Rommens PM. Kann die proximale Tibiafraktur genagelt werden? Der Einfluss des Designs auf die klinische und biomechanische Leistungsfähigkeit eines neuen intramedullären Nagels. Akt Traumatol 2003; 33: $69-75$

${ }^{9}$ Hessmann MH, Blum J, Hofmann A, Küchle $\mathrm{R}$, Rommens PM. Internal Fixation of Proximal Humeral Fractures. Current Concepts Eur J Trauma 2003; 29: 253-261

${ }^{10}$ Hessmann MH, Rommens PM. Das biomechanische Verhalten winkelstabiler Implantatsysteme am proximalen Humerus Verlag Hans Huber, Bern, Göttingen,Toronto, Seattle 2003

${ }^{11}$ Ito K, Grass R, Zwipp H. Internal fixation of supracondylar femoral fractures: comparative biomechanical performance of the 95degree blade plate and two retrograde nails. J Orthop Trauma 1998; 12: 259-266

12 Ito K, Hungerbühler R, Wahl D, Grass R. Improved intramedullary nail interlocking in osteoporotic bone. J Orthop Trauma 2001; 15: $192-196$

${ }^{13}$ Janzing HMJ, Stockman B, Van Damme G, Rommens P, Broos PLO. The retrograde intramedullary supracondylar nail: an alternative in the treatment of distal femoral fractures in the elderly? Arch Orthop Trauma Surg 1998; 118: 92 - 95

${ }^{14}$ Lill H, Hepp P, Korner J, Kassi JP, Verheyden AP, Josten C, Duda GN. Proximal humeral 

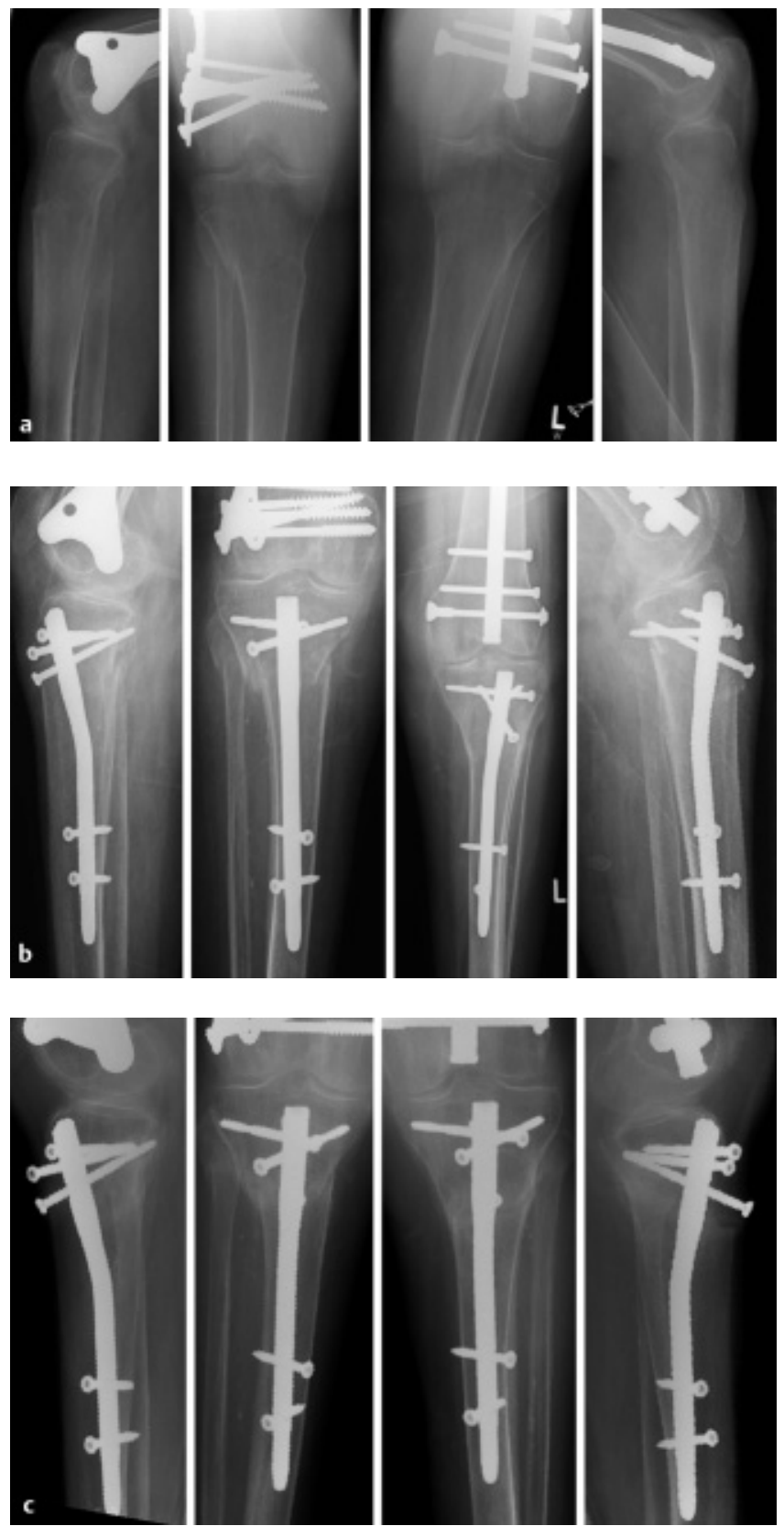

Abb.19 (a) Beidseitige proximale metaphysäre Tibiafrakturen bei einer 91-jährigen Patientin mit erheblicher Osteoporose. (b) Beidseitige Behandlung mit zwei PTN, jeweils proximal winkelstabil verriegelt, postoperativ. (c) Röntgenaufnahmen 8 Monate nach Operation mit Ausheilung der Fraktur. fractures: how stiff should an implant be? A comparative mechanical study with new implants in human specimens. Arch Orthop Trauma Surg 2003; 123: 74-81

${ }^{15}$ Mittlmeier Th, Stedtfeld HW, Ewert A, Beck M, Frosch B., Gradl G. Stabilization of proximal humeral fractures with an angular and sliding stable antegrade locking nail (Targon PH). J Bone Joint Surg 2003; 85-A-S4: 136 146

${ }^{16}$ Ritter G, Biegler M, Ahlers J. Frakturheilung unter den besonderen Bedingungen einer hochstabilen Osteosynthese mit einem neuartigen Kompressionsverriegelungsmarknagel. Hefte Unfallhlkde 1987; 189: 1197-1201

${ }^{17}$ Ritter G. Biomechanische Voraussetzungen für die Kompressionsosteosynthesen mit dem neuen AO-Universal-Marknagel. Hefte Unfallhlkde 1989; 207: 304-307

${ }^{18}$ Rommens PM, Blum J, Runkel M. Retrograde nailing of humeral shaft fractures. Clin Orthop 1998; 350: 26-39

${ }^{19}$ Rommens PM, Blum J. Die retrograde Nagelung mit dem UHN Klinische Ergebnisse. Akt Traumatol 2001; 31: 125-132

${ }^{20}$ Stedtfeld HW, Attmanspacher W, Thaler K, Frosch B. Fixation von Humeruskopffrakturen mit anterograder Marknagelung. Zentralbl Chir 2003; 128: 6-11

${ }^{21}$ Walcher F, Frank J, Marzi I. Retrograde Nailing of Distal Femoral Fracture - Clear and Potential Indications. Eur J Trauma 2000; 26: $155-68$

${ }^{22}$ Weckbach A, Weißer Ch, Blattert TR. Marknagelung am Unterarm Trauma Berufskrankh 2001; 3 [Suppl 2]: S297-S302

\section{Priv.-Doz. Dr. med. Jochen Blum}

Oberarzt

Univ.-Prof. Dr. med.

Pol Maria Rommens

Klinikdirektor

Klinik und Poliklinik für Unfallchirurgie Johannes Gutenberg-Universität Mainz Langenbeckstr. 1

D-55131 Mainz 